\title{
CEBADO DE MANATÍES (TRICHECHUS MANATUS) EN VIDA LIBRE SEGÚN EXPERIMENTOS DE PREFERENCIA COMO BASE PARA LA CAPTURA MEDIANTE LA TÉCNICA DE ENCIERRO-TRAMPA, PARQUE NACIONAL TORTUGUERO, LIMÓN COSTA RICA
}

\author{
Alexander Gómez Lépiz ${ }^{1}$
}

\begin{abstract}
RESUMEN
Este estudio determinó la preferencia del manatí por algunos recursos alimenticios que constituyen su dieta en la región. La preferencia alimenticia en un herbívoro es abordada mediante "experimentos de restaurante", al dejar que los animales escojan libremente entre los alimentos ofrecidos. Con este propósito se proporcionaron las principales plantas consumidas por el manatí (Paspalum repens, Urochloa mutica, Hydrocotyle ranunculoides y Eichhornia crassipes) en el área de estudio, junto a otras plantas de consumo humano que son proporcionadas en los zoológicos como parte de su dieta (manzanas, zanahorias, lechugas y mangos). De todas las plantas ofrecidas, el pasto $P$. repens fue la planta preferida y se utilizó para evaluar con éxito una estructura preliminar de encierro-trampa en donde los manatíes ingresaron. La preferencia por esta planta podría estar relacionada con características que no fueron evaluadas durante la presente investigación como: palatabilidad, microelementos, textura.
\end{abstract}

Palabras claves: Preferencia, cebado, Trichechus manatus, Costa Rica, Parque Nacional Tortuguero.

\begin{abstract}
This study determined the preference of the manatees for some food resources, which constitute their diet in the region. The preference of the herbivore is typically shown through the "cafeteria feeding trials" technique: the animals are left to choose freely among the plants available for them. In order to evaluate the preference of the manatees through this technique, plants normally consumed by the manatee were offered (Paspalum repens, Urochloa mutica, Hydrocotyle ranunculoides y Eichhornia crassipes) in the study area, next to other vegetables normally consumed by humans, which are usually offered at a Zoo as part of their diet. Of all the plants offered, the grass P. repens was the preferred plant, which was successfully used to evaluate the preliminary structure lock up-trap where the manatees entered. The preference for this plant could be attributed to characteristics that were not evaluated for this study, such as palatability, microelements, and/or texture.
\end{abstract}

Keywords: Preference, baiting, Trichechus manatus, Costa Rica, Tortuguero National Park.

\section{INTRODUCCIÓN}

Los manatíes del Caribe (Trichechus manatus Linnaeus, 1978) se mueven libremente entre ambientes marinos y de agua dulce, alimentándose de una amplia variedad de vegetación sumergida, flotante y emergente (Best, 1981). De esa variedad de plantas algunas son consumidas con mayor frecuencia, lo que evidencia procesos de selección por parte de los manatíes hacia algunas de esas plantas. 1 Instituto Internacional en Conservación y Manejo de Vida Silvestre, Universidad Nacional, Apartado 1350-300,
Heredia, Costa Rica. gmanatusl@gmail.com

Recibido 29-III-2010

Aceptado 04-VIII-2010

DOI: http://dx.doi.org/10.15359/revmar.2.9 
La preferencia en la dieta de un animal se asocia con aquellos alimentos que le brindan nutrientes (Frazer y Scott, 2002). En general, los herbívoros no reaccionan de forma indiscriminada ante el ofrecimiento de diferentes follajes (Janzen, 1982). Por ello, estos animales aumentan el consumo de aquellas plantas que les producen beneficios nutricionales y limitan el consumo de aquellas que les son tóxicas o de baja calidad para su dieta, por lo tanto, la preferencia o la aversión resultan en parte por las consecuencias digestivas que provocan las plantas consumidas (Launchbaugh et al. 2001).

Litvaitis et al. (1994) señalan que la preferencia es valorada mediante los "experimentos de restaurante", donde se les ofrece a uno o varios animales la opción de alimentarse de varios ítems de forma indiscriminada, donde finalmente se valora la preferencia por aquellos que escogen con mayor frecuencia. La información relacionada con la dieta del manatí en Costa Rica se ha asociado a registros de observaciones de plantas encontradas en sitios de alimentación y a reportes de los habitantes de las zonas donde se localiza la especie (Reynolds et al. 1995; Jiménez, 1999; Smethurst y Nietschmann, 1999).

En general, la información relacionada con el manatí en Costa Rica es limitada, y para ello, se han realizado dos intentos de captura con redes para colocarles radio marcas y así desarrollar estudios con telemetría, para reducir esos vacíos de información. A pesar de estos esfuerzos, en ambas ocasiones, los intentos han sido fallidos (Jiménez, 2005). Ambos procesos refuerzan la idea de captura mediante el cebado con alimentos que forman parte de la dieta natural de la especie y con alimentos proporcionados en zoológicos como forma de favorecer su captura.
La opción de trampeo mediante encierro-trampa, basada en el cebado de manatíes, se ha implementado de forma exitosa en África, como técnica para la captura de esta especie. La técnica consiste en el trampeo mediante una estructura de encierro, donde el manatí activa la trampa mediante un dispositivo automático (Reynolds y Odell, 1991). Con la intención de darles continuidad a los esfuerzos relacionados con la conservación de los manatíes en el territorio costarricense, esta investigación tuvo por objetivos: i) identificar la preferencia del manatí hacia plantas acuáticas (emergentes y flotantes) y plantas cultivadas por humanos para utilizarlas en pruebas de cebado con la especie en vida libre, ii) evaluar la preferencia cuantitativa de cada planta ofrecida, iii) evaluar la opción de alimentar a los manatíes sobre un mismo sitio en una estructura preliminar de encierro.

\section{ÁREA DE ESTUDIO}

El estudio se desarrolló en el Parque Nacional Tortuguero (PNT), en la Provincia de Limón, Costa Rica. El sitio donde se realizaron los experimentos fue en el caño conocido como "Aguas Frías", que se ubica a $2 \mathrm{~km}$ hacia el sur del puesto principal de guardaparques del PNT. El sitio de experimentación pertenece a un área restringida del PNT, por lo que el acceso a este sector es limitado. El turismo que ingresa a esta área lo hace bajo ciertas regulaciones como: ingreso con un guía de turismo oficial y limitaciones en la velocidad de recorrido sobre esta $(5 \mathrm{~km}$ por hora aproximadamente). En esta zona desemboca el río Tortuguero, que se une con los principales canales turísticos del parque nacional.

La vegetación acuática en los primeros kilómetros sobre este sector está dominada 
Cuadro 1. Fases de desarrollo para el cebado de los manatíes en la estructura preliminar de encierro-trampa

Table 1. Development stages for manatees baiting in the preliminary structure enclosure

\begin{tabular}{llc}
\hline \multicolumn{1}{c}{ Fase } & \multicolumn{1}{c}{ Descripción } & Duración \\
\hline $\begin{array}{l}\text { I- Evaluación de la reacción } \\
\text { ante los diferentes alimentos }\end{array}$ & $\begin{array}{l}\text { Evaluación de la reacción de los manatíes } \\
\text { ante el ofrecimiento de diferentes tipos de } \\
\text { plantas propios del área de estudio. }\end{array}$ & 4 meses \\
II- Cebado & $\begin{array}{l}\text { Escogencia de plantas para evaluar e } \\
\text { implementar el cebado con los manatíes. }\end{array}$ & 5 meses \\
III- Reacción ante la & $\begin{array}{l}\text { Evaluación de la reacción de los manatíes ante } \\
\text { el ofrecimiento de P. repens (cebo) dentro de } \\
\text { estructura de encierro-trampa }\end{array}$ & 2 meses \\
& una estructura preliminar de encierro. & \\
\hline
\end{tabular}

por Eichhornia crassipes (Mart.) Solms y en algunas partes se pueden encontrar algunos parches de Urochloa mutica (Forsk.) Nguyen. Hacia adentro, sobre el río Tortuguero, se encuentran áreas dominadas por Paspalum repens P. j. Bergius.

Las aguas en este sector del PNT son de poca visibilidad en apariencia, debido al arrastre de sedimento provocado por las corrientes del río Tortuguero. La profundidad del canal en los sitios donde se desarrollaron los experimentos varía desde 50 $\mathrm{cm}$ en las orillas hasta $4 \mathrm{~m}$.

\section{MATERIALES Y MÉTODOS}

El estudio se desarrolló entre mayo del 2005 y abril del 2006 y comprendió tres fases: i) evaluación de la reacción ante los diferentes alimentos, ii) cebado, iii) reacción ante la estructura de encierro-trampa (Cuadro 1).

\section{Condiciones generales del sitio de prueba}

Los alimentos se amarraron mediante una cuerda, la cual estaba sujeta a dos estacas extendidas de forma paralela a la orilla del canal y dicha cuerda tenía una extensión aproximadamente de $45 \mathrm{~m}$. Todos los alimentos fueron sujetados de cuerdas de $90 \mathrm{~cm}$ (porciones de plantas: amarra- dos y frutos: orificios para sujetarlas y amarradas), de forma que permanecieran flotando sobre la superficie del agua y perpendiculares a la cuerda principal.

La cuerda se dividió en dos secciones, ambas con alimentos propios de la dieta del manatí en el área (pastos y lirios) (Cuadro 2). Ambas secciones de la cuerda también tenían las mismas plantas y en cantidades iguales, con el fin de determinar si hubo evidencia de preferencia por la cantidad de alimento ofrecido. En una de las secciones se colocaron alimentos de consumo humano, con el propósito de valorar si existía preferencia por estos alimentos sobre las plantas consumidas por el manatí en el área de estudio. Los alimentos de consumo humano presentados fueron: Malus silvestris (manzana), Lactusa sativa (lechuga), Daucus carota (zanahoria) y Mangifer indica (mango). Cada sección del mecate contenía: a) una porción de $4 \mathrm{~kg}$ de cada especie de planta propia de la zona; b) una porción de $2 \mathrm{~kg}$ de cada especie propia de la zona; c) una porción de $1 / 2 \mathrm{~kg}$ de cada especie propia de la zona. En la sección del mecate con alimentos de consumo humano se colocaron los alimentos como sigue: junto a las porciones de $4 \mathrm{~kg}$ : 6 manzanas, 6 lechugas, 6 zanahorias y 6 mangos; junto a las 
porciones de $2 \mathrm{~kg}$ : 3 manzanas, 3 lechugas, 3 zanahorias y 3 mangos; junto a las porciones de $1 / 2 \mathrm{~kg}$ : 1 manzana, 1 lechuga, 1 zanahoria y 1 mango.

\section{Plantas ofrecidas y cambio de estas}

De los resultados obtenidos por Gómez (2007), fueron identificadas cuatro plantas que consumen los manatíes con mayor frecuencia en la zona; dos tipos de pastos: $U$. mutica y $P$. repens; y dos especies de lirios: E. crassipes y Hydrocotyle ranunculoides L. f. Las plantas ofrecidas se cambiaron cada tres días por muestras frescas. Los alimentos fueron recolectados durante las mañanas del día que se realizaba el cambio para suministrárselos frescos.

\section{Registro de los datos}

Se llevó un registro de las ocasiones de consumo que se observaban en cada una de las porciones de alimento ofrecido. Cada ocasión de consumo fue definida como: el ramoneo total o parcial por parte del manatí o manatíes en cualesquiera de las porciones de alimento ofrecido.

Los manatíes en el área de estudio son sensibles a cualquier perturbación, y huyen con facilidad ante situaciones de amenaza, lo que dificulta la observación directa de estos. Ante tal situación, se utilizaron los rastros dejados por manatíes para desarrollar el presente estudio sobre hábitos alimenticios, según lo definido por Aranda (2000) para la observación indirecta de mamíferos como opción de desarrollo de estudios sistemáticos. Gómez (2007) indica que un rastro fresco dejado por los manatíes al alimentarse de plantas emergentes o flotantes, no puede confundirse con el rastro dejado por otro herbívoro, debido a la cantidad de alimento que estos animales consumen y el tipo de corte posterior al ramoneo, por lo que ambos criterios constituyeron evidencias suficientes para demostrar la presencia de manatíes en un determinado lugar.

\section{Pruebas de cebado y estructura de encierro}

En una primera fase del trabajo de campo, se ofrecieron las plantas propias de la dieta del manatí para el área de estudio y así determinar la diferencia en el consumo de las plantas ofrecidas e identificar sus patrones de consumo. Después se desarrolló una fase de cinco meses de cebado solo con $P$. repens y, por último, durante dos meses se evaluó el ingreso de los manatíes a una estructura preliminar de encierro (Cuadro 2).

La estructura de encierro fue construida con estacas de Gynerium sagittatum colocadas a manera de círculo, el cual tenía un espacio para el ingreso y salida de los manatíes a la estructura. El diámetro del encierro fue de $8 \mathrm{~m}$ y fueron necesarias 200 estacas para darle la forma deseada a la estructura final, estas estacas fueron reforzadas con mecate para sujetarlas.

Las porciones de $P$. repens utilizadas para el cebado dentro del encierro se colocaron en el centro de la estructura y se llevó un registro de las ocasiones en que estas fueron consumidas.

\section{Análisis estadístico}

Se utilizó una prueba de Bondad de Ajuste (Sokal y Rohlf, 1979) para determinar la diferencia en la cantidad de ocasiones de consumo en que los manatíes se alimentaron de las distintas especies de plantas colocadas en los "experimentos de restaurante" y el patrón de consumo de estas plantas. Se utilizó el programa Statgraphics Centurium XV (Manugistics, 2005) para desarrollar una tabla de contingencia, según los planteamientos de Sokal y Rohlf (1979). 
Cuadro 2. Distribución de los alimentos presentados a los manatíes en el lugar de evaluación de los "experimentos de restaurante"

Table 2. Distribution of food presented to manatees in the evaluating place for the "cafeteria feeding trials"

\begin{tabular}{cl}
\hline Distancia (m) & \multicolumn{1}{c}{ Alimentos colocados } \\
\hline 0 & Sin alimentos \\
5 & Porciones de $4 \mathrm{~kg}$ de plantas presentes en la dieta del manatí \\
10 & Porciones de $2 \mathrm{~kg}$ de plantas presentes en la dieta del manatí \\
15 & Porciones de $1 / 2 \mathrm{~kg}$ de plantas presentes en la dieta del manatí \\
20 & Sin alimentos \\
25 & Sin alimentos \\
30 & $\begin{array}{l}\text { Porciones de } 1 / 2 \mathrm{~kg} \text { de plantas presentes en la dieta del manatí junto a } 1 \\
\text { unidad de cada planta de consumo humano }\end{array}$ \\
35 & $\begin{array}{l}\text { Porciones de } 2 \mathrm{~kg} \text { de plantas presentes en la dieta del manatí junto a } 3 \\
\text { unidades de cada planta de consumo humano }\end{array}$ \\
40 & $\begin{array}{l}\text { Porciones de 4 kg de plantas presentes en la dieta del manatí junto a } 6 \\
\text { unidades de cada planta de consumo humano } \\
\end{array}$ \\
& Sin alimentos
\end{tabular}

\section{RESULTADOS}

No hubo evidencia de que los manatíes se alimentaron de los productos de consumo humano (manzanas, lechugas, mangos y zanahorias). En cuanto a las plantas consideradas como parte de la dieta regional de los manatíes, solo en pocas ocasiones (una ocasión corresponde a dos días, mientras se exponía el alimento y se realizaba el cambio a porciones frescas) se alimentaron de estas, a excepción de $P$. repens. Las plantas fueron ofrecidas juntas en 52 ocasiones y durante las últimas 36 ocasiones, los manatíes se alimentaron de forma ininterrumpida y en todas las ocasiones se consumieron $P$. repens (Fig. 1), lo que marcó la diferencia $\left(X^{2}=80.692\right.$, g.l $\left.=3, P<0.005\right)$ con el resto de especies ofrecidas (U. mutica, E. crassipes y $H$. ranunculoides) que solo fueron consumidas de una a tres veces. El patrón de consumo de $P$. repens se mantuvo a lo largo del tiempo en que consumió esta especie $\left(X^{2}=22.61, g . l=30, P<0.831\right)$, en relación con las otras plantas ofrecidas. El tiempo en que los manatíes se alimentaron de $P$. repens se dividió en períodos de 10 días, para comparar el patrón de consumo de esta planta con respecto al resto de plantas colocadas.

La preferencia por el tamaño de las porciones de alimento ofrecidas mostró que los manatíes se alimentaron de todos los grupos de $P$. repens que fueron colocados, sin discriminar su peso.

Durante la última fase, que correspondió a las evaluaciones para la estructura de encierro, los manatíes ingresaron a dicha estructura en el $92.8 \%$ de todas las ocasiones posibles (24 registros de consumo dentro del encierro, de un total de 26 ocasiones en que se realizaron observaciones).

\section{DISCUSIÓN}

El concepto de preferencia de una especie por uno o más recursos es definido de forma similar en los textos en que se 


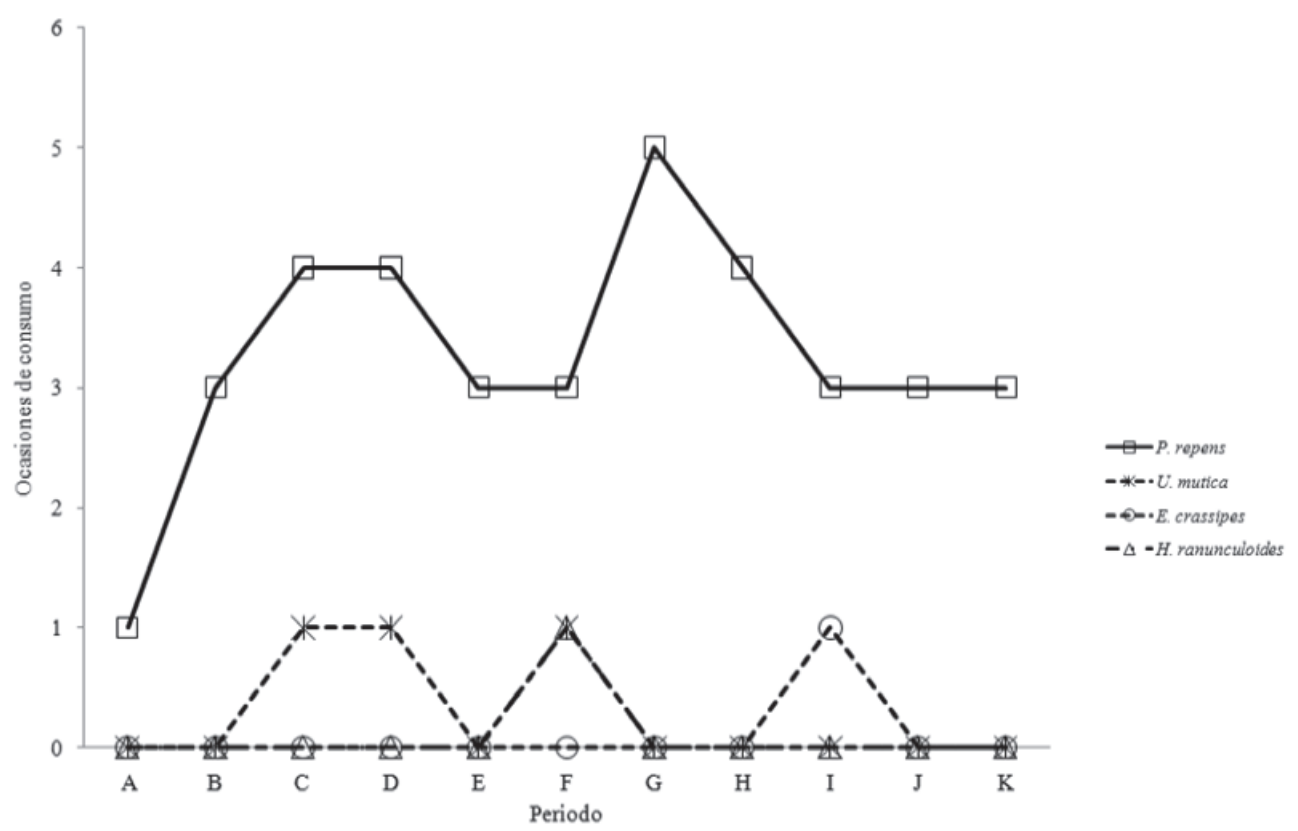

Fig. 1. Períodos de 10 días para las ocasiones de consumo por manatíes sobre las plantas ofrecidas durante los "experimentos de restaurante". Plantas colocadas: gamalote (Paspalum repens), pará (Urochloa mutica), choreja (Eichhornia crassipes), comalillo (Hydrocotyle ranunculoides)

Fig.1. Ten days periods for the occasions of consumption by manatees on plants placed in the "cafeteria feeding trials". Plants placed: gamalote (Paspalum repens), pará (Urochloa mutica), choreja (Eichhornia crassipes), comalillo (Hydrocotyle ranunculoides)

enfoca el tema de la selección de recursos. Litvaitis et al. (1994) y Johnson (1980) definen la "preferencia", como la posibilidad de que un recurso pueda ser seleccionado por una especie, si le es ofrecido en cantidades iguales junto a otras especies. Los pastos han constituido una parte importante en la dieta de los manatíes (O'Shea, 1994). En particular, esta investigación evidenció que $P$. repens fue la planta preferida por estos animales.

Este pasto acuático constituiría un recurso importante para los manatíes en los sistemas de agua dulce del PNT. Según lo observado por Gómez (2007), estos animales se alimentaron de forma natural en su mayoría de U. mutica; sin embargo, los "experimentos de restaurante" en este estudio permitieron descartar esta planta como preferida (U. mutica).

Colares y Colares (2002) resaltan la importancia de $P$. repens en la dieta de otro sirenio: el manatí amazónico (Trichechus inunguis Natterer, 1883) (con un sistema digestivo similar al del T. manatus) y señalan cómo este animal muestra una preferencia específica de consumo por $P$. repens, ya que registraron esta planta como la especie con la mayor frecuencia de aparición en las heces analizadas del manatí amazónico. Además, estos autores también registraron que un manatí amazónico cautivo no se alimentó de otras plantas que le fueron ofrecidas hasta consumir la totalidad de las muestras de $P$. repens. 
Los manatíes tienen un sistema digestivo adaptado para aprovechar la celulosa de las plantas que consumen de manera eficiente, a diferencia de otros herbívoros que poseen un sistema digestivo similar (Burn, 1986). El lento pasaje de los alimentos por el tracto digestivo de los manatíes (hasta 146 horas) (Burn, 1986) les permite que puedan consumir alimentos considerados para los herbívoros como de "baja calidad" (con altos contenidos de fibra y bajos contenidos de proteína) y que esos alimentos puedan soportar todo ese trayecto a través del sistema digestivo (Smith, 1993; Reynolds y Rommel, 1996; Rommel et al. 2003).

Las evaluaciones nutricionales realizadas de $P$. repens señalan que este pasto posee altos valores de fibra y bajo contenido de proteína (Gómez, 2007). Sin embargo, se puede considerar que otras características propias de la planta (textura, palatabilidad, microelementos) podrían otorgarle la condición de preferencia para los manatíes, en relación con otras plantas que son parte de su dieta en la zona.

Con las observaciones de preferencia de los manatíes ante $P$. repens, en este estudio se abren nuevas posibilidades de captura para esta especie en Costa Rica, ya que los manatíes ingresaron a la estructura preliminar de encierro en la mayoría de las ocasiones en que se colocó este pasto.

El método de captura de mayor funcionalidad de manatíes ha sido mediante redes (Deutsch et al. 2003). A pesar de ello, este sistema en Costa Rica fue implementado en dos ocasiones sin éxito (Jiménez, 2005), y como opción se inicia con las pruebas de cebado como metodología alternativa para su captura.

El panorama que se presenta para la captura de manatíes mediante el sistema de encierro-trampa parece ser el más adecuado, ante la imposibilidad de capturar- los con los métodos tradicionales para la especie (Reynolds y Odell, 1991), según las características del hábitat del manatí en la zona de estudio. El encierro-trampa tiende a ser funcional, ya que los manatíes ingresan a este sin ningún problema para consumir las cantidades de $P$. repens que les son ofrecidas. Es evidente que el cebo más adecuado para intentar la captura es el $P$. repens y queda por delante desarrollar un proceso en el que se pueda utilizar algún sistema de trampa para que el manatí lo active de forma automática.

\section{AGRADECIMIENTOS}

A la Fundación Rufford de Inglaterra, por el financiamiento mayoritario del desarrollo de la etapa de campo de este trabajo; al Instituto Internacional en Conservación y Manejo de Vida Silvestre y a Jorge Fallas, por facilitar aspectos logísticos y académicos para el desarrollo de esta investigación; al Área de Conservación Tortuguero, por el apoyo de estadía parcial en la etapa de toma de datos; al Hotel Manatus, a Fernando y Lili Figuls, por el apoyo incondicional para facilitar y fomentar la investigación de manatíes en Costa Rica; a Ignacio Jiménez Pérez, por las observaciones y los comentarios oportunos para la elaboración del presente documento; a Michael McCoy Bolton, por la orientación y motivación para el desarrollo adecuado en etapas fundamentales de este trabajo; a James Powell, Robert Bonde y Lucy Keith, por ayudar a dar forma a la idea básica que da sustento a esta investigación; a Arilyn Pinnock, José Luis Alvarado, Heidy Saborío Salas y Daryl Loth, porque fueron un apoyo fundamental durante diferentes etapas de este trabajo; a la Fundación Salvemos al Manatí de Costa Rica, por el préstamo de equipo fundamental para el traslado dentro del área de estudio. 


\section{BIBLIOGRAFÍA}

Aranda, M. (2000). Huellas y otros rastros de los mamíferos grandes y medianos de México. Xalapa, México: Instituto de Ecología, A. C.

Best, R. (1981). Foods and Feeding Habits of Wild and Captive Sirenia. Mamm. Rev., 11, 3-29.

Burn, D. (1986). The Digestive Strategy and Eficiency of the West Indian Manatee, Trichechus manatus. Bioch. Phys., 85A (1), 139-142.

Colares, I. \& Colares, E. (2002). Food Plants Eaten by Amazonian Manatee (Trichechus inunguis, Mammalia: Sirenia). Braz. Arch. of Biol. and Tech., 45 (1), 67-72.

Deutsch, Ch., Reid, J., Bonde, R., Easton, D., Kochman, H. \& O'Shea, T. (2003). Seasonal Movements, Migratory Behavior, and Site Fidelity of West Indian Manatee Along the Atlantic Coast of the United States. Wild. Mongr., 67 (1), 1-77.

Frazer, K. \& Scott, M. (2002). Determinants of Dietary Preference in Yellow-Rumperd Warblers. Wilson Bull., 114 (2), 243-248.

Gómez, A. (2007). Uso y Selección de Plantas Emergentes y Flotantes en la DietadelManatí(Familia:Trichechidae: Trichechus manatus) en el Parque Nacional Tortuguero, Limón, Costa Rica. Tesis de Maestría no publicada. Instituto Internacional en Conservación y Manejo de Vida Silvestre. Universidad Nacional, Heredia, Costa Rica.

Janzen, D. (1982). Wild Plant Acceptability to a captive Costa Rican Baird's Tapir. Brenesia, 19, 99-128

Johnson, D. (1980). The Comparison of Usage and Availability Measurements for Evaluating Resuourses Preferente. Ecol., 61 (1), 65-71.

Jiménez, I. (1999). Estado de Conservación, Ecología y Conocimiento Popular del Manatí (Trichechus manatus) en Costa Rica. Vida Silv. Neotr., 8 (12), 18-30.

Jiménez, I. (2005). Informe Final de las Campañas de Captura y Marcaje de Manaties en el Parque Nacional Tortuguero. Costa Rica: EUNA.

Launchbaugh, K., Provenza, F. \& Pfister, J. (2001). Herbivore Response to Anto-quality Factors in Forages. J. Range Manag., 54, 431-440.

Litvaitis, J., Titus, K. \& Anderson, E. (1994). Measuring Vertebrate Use of Terrestrial Habitats and Food. En T. A. Bookhout (Ed.), Research and Management Techniques for Wildlife and Habitat (pp. 254-274). U.S. Fish and Wildlife Service. Maryland: The Wildlife Society.

Manugistics, Statgraphics Centurium, XV. (2005). Launching the program, and creating a simple data file [Computer program manual]. Estados Unidos: StatPoint Technologies, Inc.

O'Shea, T. J. (1994). Manatees. Sci. Amer., 66-72.

Reynolds, J., Szelistowski, W. \& León, M. (1995). Status and Conservation of Manatee Trichechus manatus manatus in Costa Rica. Biol. Conser, 71, 193-196.

Reynolds, J. \& Odell, D. (1991). Manatees and Dugongs. Estados Unidos: Library of Congreso in Publication Data. Reynolds, J. \& Rommel, S. (1996). Structure and Function of the Gastrointestinal Tract of the Florida Manatee, Trichechus manatus latirostris. Anat. Record., 245, 539-558. 
Rommel, S., Reynolds, J. \& Lynch, H. (2003 octubre). Adaptations of Herbivorous Marines Mammals. Ponencia presentada en el VI International Symposium on the Nutrition of Herbivores. Proceedings of an International Symposium held in Mérida, México.

Smethurst, D. \& Nietschmann, B. (1999). The Distribution of Manatees (Trichechus manatus) in the Coastal Waterways of Tortuguero, Costa Rica. Biol. Conser., 89, 267-274.
Smith, K. (1993). Manatee Habitat and Human-related Threats to Seagrass in Florida: A review. Departamento de Protección al Medio Ambiente. Florida, Estados Unidos: Editorial Division of Marine Resources.

Sokal, R. \& Rohlf, J. (1979). Biometría: Principios y métodos estadísticos en la investigación biológica. Madrid, España: Ediciones H. Blume. 